\title{
Produktionsplanungs- und -steuerungssysteme (PPS) in der internen und externen Logistik
}

\author{
Prof. Dr. oec. László Ungvári
}

Unter PPS (Produktionsplanung und -steuerung) wird die Gesamtheit aller Dispositionen zusammengefaßt, welche auf die Festlegung eines Ansatz- bzw. Produktionsprogramms und auf die Organisation des mengenmäßigen und zeitlichen Vollzugs diesen Programms gerichtet sind. Die PPS umfaßt also die gesamt Auftragsabwicklung von der Angebotsbearbeitung bis zum Versand. Dabei kommt sie sowohl mit externen Organisationen z. B. über den Einkauf und den Versand als auch mit internen betrieblichen Abteilungen wie Konstruktion, Teilfertigung und Montage in Berührung.

Die Produktionsplanung und -steuerung ist ein wichtiger Bestandteil der Unternehmenslogistik, da die Logistik in einem Industrieunternehmen die Planung, Durchführung, Steuerung, Kontrolle des Materialflusses und des zugehörigen Informationsflusses von der Beschaffung über die Produktion bis zur Entsorgung und Distribution umfaßt. Daher ist es naheliegend, daß man heute mit dem Kïrzel PPS nahezu in jedem Fall die Erwartung nach einem Softwarepaket - und nicht selten - mit allen Funktionalitäten verbindet, die externe und interne Aspelte der Unternehmenslogistik erfassen. Im Bild 1 sind die internen und externen Bereiche der Logistik in einem Industrieunternehmen dargestellt.

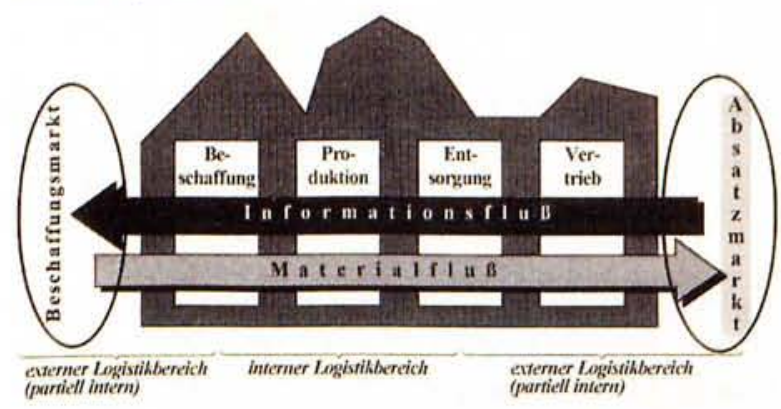

Bild 1: Interdependenzen zwischen internen und externen Logistikbereichen in Industrieunternehmen

Das obige Bild 1 macht zugleich auch die Gleichnisse und die Unterschiede zwischen Logistik und PPS deut-

\begin{tabular}{|l|l|l|}
\cline { 2 - 3 } \multicolumn{1}{c|}{} & Informationsfluß & Materialfluß \\
\hline Beschaffung & PPS & \\
\hline Produktion & & \\
\hline Entsorgung & & \\
\hline Vertrieb & & \\
\hline
\end{tabular}

Bild 2: Stellung der PPS in der Logistikmatrix lich. Während in der Logistik die Betrachtungen sowohl dem Informations- als auch dem Materialfluß gewidmet werden, wird in einer PPS-Lösung in der Hauptsache der Informationsfluß abgebildet. Die OOS ist zwar nicht die Logistik, die PPS stellt aber innerhalb der Logistik eine wesentliche Komponente dar. In der nachstehenden Logistikmatrix (Bild 2) soll dies unterstrichen werden. Auf dem kaum noch ïberschaubaren PPS-Markt in der Bundesrepublik tummeln sich PPS-Lösungen, die sich trotz ihrer relativ kurzen Geschichte in vier bzw. fünf Entwicklungetappen einordnen lassen (vgl. Bild 3).

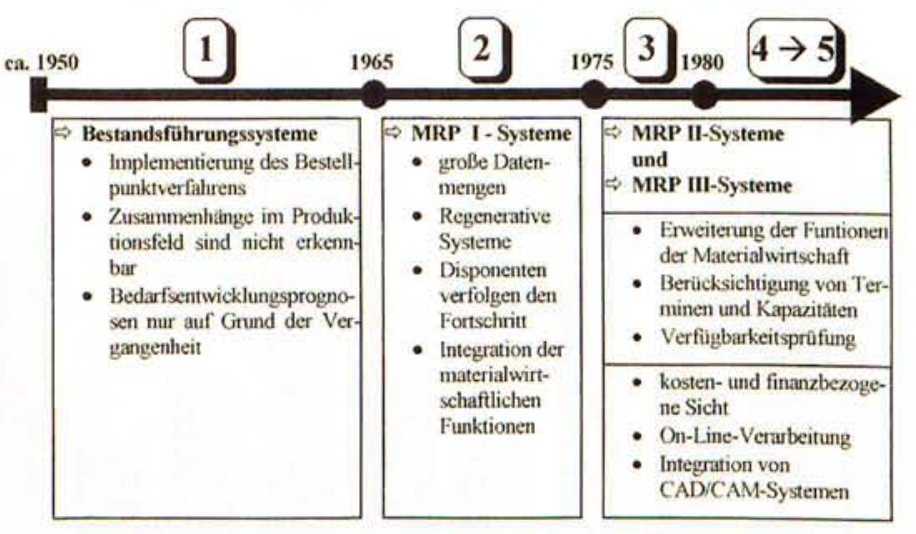

Bild 3: Entwicklungsgeschichte von PPS-Systemen

In der ersten Entwicklungsetappe handelt es sich im Grunde noch gar nicht um PPS-Lösungen im heutigen Sinn. Die ersten Implementierungen in Form von Bestandsführungssystemen waren eher Systeme für den Handel oder/und für den materialwirtschaftlichen Bereich industrieller Unternehmen. Die ersten Lösungen nach dem sukzessiven Prinzip stammen aus der Zeit nach 1965. Dies waren zwar auch Systeme „nur“ für den Bereich Materialwirtschaft, jedoch ist hervorzuheben, daß in diesen Lösungen mit der Integration der einzelnen materialwirtschaftlichen Funktionen (Material-Requirement-Planning - MRP I) bereits begonnen wurde.

In den Abkürzungen MRP II stehen die Buchstaben M, $R$ und $P$ für Manufactoring-Resource-Planning und in MRP III Management-Resource-Planning. Der Unterschied zwischen MRP II und MRP III besteht dabei darin, daß die mengen- und zeitbezogene Sicht der MRP IISysteme in den MRP III-Systemen um die kosten- und finanzbezogene Sicht erweitert wurde. In diesem $\mathrm{Zu}$ sammenhang wird heute zunehmend von ERP-Systemen (Enterprise-Resource-Planning) gesprochen. Die MRP IISysteme lassen die Bildung von Losgrößen auf jeder Dispositionsstufe zu, schreiben aber keine feste Losgrößenphilosophie vor. Die Losgrößen werden dann in Kapazitätsbedarfe (Modul Zeitwirtschaft/Kapazitätsbedarf) umgerechnet und mit den Kapazitätsangeboten 
verglichen. um eine hinreichende Sicherheit des Produktionsablaufs zu gewährleisten. Die Rückmeldeinformationen über die aktuellen Ablaufsdaten und Beschaffungssituationen werden mit dem Produktionsplan verglichen, um Abweichungen (Ruickstände) festzustellen. Auf der Grundlage dieser Informationen können dann Prioritäten in der Fertigung und im Einkauf festgelegt werden. MRP-Systeme (MRP II aufwärts) sind auch in der Lage, Berichte für das Management zur Koordinierung unterschiedlicher Funktionsbereiche zu erstellen. In modernen MRP-Systemen können auch Simulationsberechnungen durchgefuihrt werden, damit die Auswirkungen von Variantenlösungen getestet werden können. Am häu-

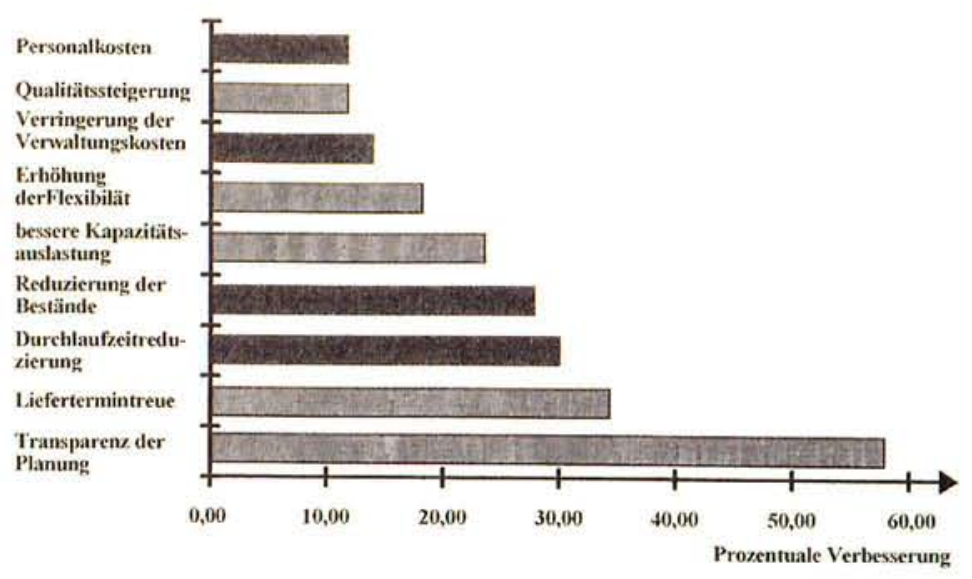

Bild 5: Nutzeffekte durch Einführung von PPS-Systemen (Unternehmensbefragung) figsten geht es dabei um die Betrachtung des Produktionsplanes in Abhängigkeit von den Ressourcenverfügbarkeiten.

liegender Kundenaufträge fertigen. Diese Auftragsfertiger können auf dem zunehmend härter werdenden Konkurrenzmarkt nur bestehen, wenn sie die Vorstellungen ihrer Kunden weitestge-

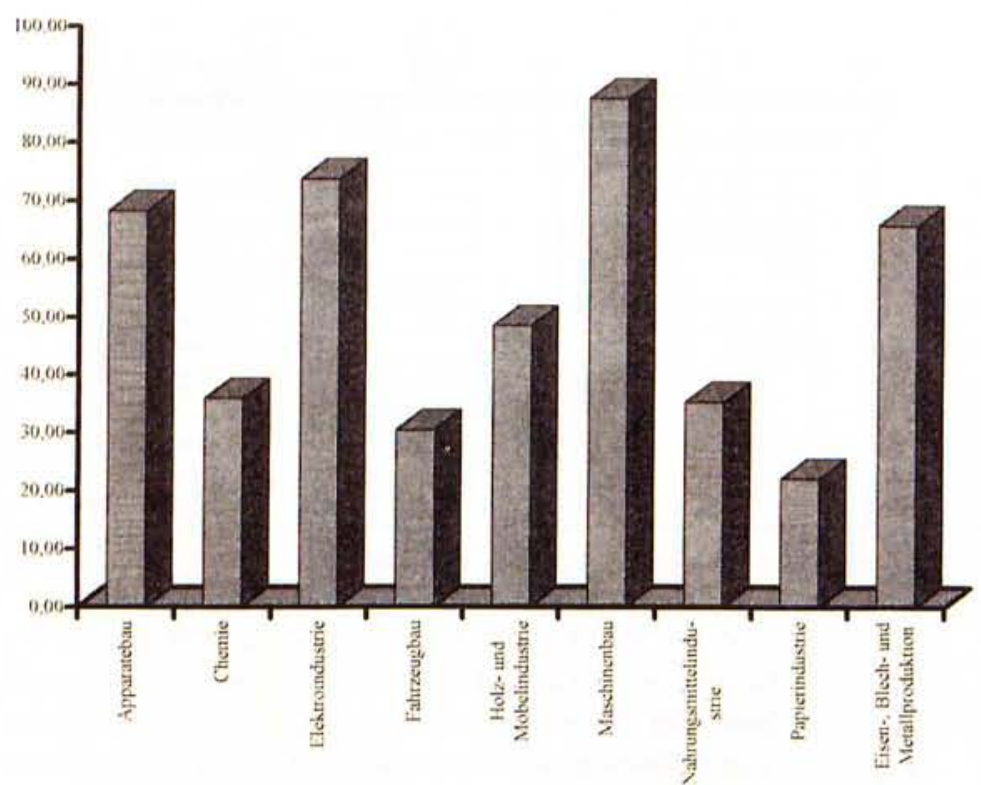

Bild 4: Verbreitung von PPS-Systemen in der Bundesrepublik Deutschland nach Branchen hend berïcksichtigen.

Die geschilderte Tatsache hat natürlich auch Konsequenzen für die Anforderungen dieser Fertigungsprozesse an die PPS-Systeme. In solchen Unternehmen entsteht der weit überwiegende Anteil der Tätigkeiten in der operativen Produktionsplanung aufgrund von Kundenaufträgen, Hier steht der individuelle Kundenwunsch hinsichtlich Zeit, Menge und auch Produktgestaltung im Mittelpunkt. Die traditionelle Philosophie der Sukzessivplanung von MRP-Systemen erweist sich in der Auftragsproduktion als hinderlich und unpraktikabel.

Im Bild 6 sind verschiedene Ausprägungsformen der Auftragsfertigung mit Beschreibungen bezüglich der Beherrschbarkeit durch ein konventionelles PPS-System dargelegt. Die Numerierung der Fälle 1 bis 4 ent-

Die Verbreitung von PPS-Systemen hängt wie die aus dem Bild 4 deutlich stark von der vorherrschenden Fertigungsart in den Unternehmen ab.

Der Einsatz von PPS-Systemen in den Unternehmen ist vorrangig durch die erzielbaren Nutzeffekte wie

- höhere Lieferbereitschaft bei den Komponenten (Lager),

- bessere Termintreue,

- kürzere Auftragsdurchlaufzeiten,

- geringere Lagerbestände,

- höhere Kapazitätsauslastung

sinnvoll und häufig auch unerläßlich.

Das Bild 5 stellt die nach Meinung der Unternehmen durch eine PPS-Einfuihrung tatsächlich erzielten Nutzeffekte in Form prozentualer Verbesserungen dar.

In der Bundesrepublik gibt es eine Vielzahl von Unternehmen, welche ihre Produktarten nicht auf Vorrat, $d$. h. zunächst kundenanonym, sondern auf der Basis vorspricht der Stufung hinsichtlich ihrer Handhabbarkeit mittels konventioneller PPS-Systeme. Während die Gruppe 1 noch relativ gut disponiert werden kann, versagen dagegen beider Gruppe 4 die konventionellen PPS-Lösungen gänzlich.

Diese Fälle, insbesondere die Gruppen 3 und 4 im Bild 6 verlangen eine neue PPS-Philosophie von PPS als die der Sukzessivplanung. Da das Produkt- und somit auch das Produktionsprogramm eines Auftragsfertigers nicht vorgegeben werden kann, gibt es auch keine Grunddaten in dem Sinn wie bei der Serien- oder Massenfertigung. Dem Auftragsfertiger stehen Stücklisten und Arbeitspläne häufig nicht zur Verfügung, da die genaue Produktdefinition erst mit dem Kundenauftrag erfolgt. Geht man bei bestimmten Kundenaufträgen davon aus, daß diese einmalige Aufträge sein dürften, so werden auch keine Zeichnungen, Stücklisten und Arbeitspläne angelegt. Das Fehlen wichtiger Informationen erschwert natïrlich die Produktionsplanung und -steuerung sehr. 
In diesem Fall ist es auch sehr kompliziert, über die Annahme oder Ablehnung eines Kundenauftrages zu entscheiden, denn diese Entscheidungen sollen entsprechend der betriebswirtschaftlichen Theorie aufgrund von Deckungsbeiträgen geschehen. diese Entscheidungen setzen allerdings zuverlässige Vorkalkulationen voraus, diese benötigen wiederum das Vorhandensein von genauen Verbrauchsdaten. Die exakte Ermittlung von Verbrauchsdaten kann nur aufgrund von Stücklisten durchgefuihrt werden.

Ähnliche Schwierigkeiten gibt es in der Kapazitätswirtschaft. Da Arbeitspläne für die Kundenaufträge fehlen, kann insbesondere die Kapazitätsbedarfsplanung keineswegs zuverlässig durchgefuihrt werden.

Seit Anfang der achtziger Jahre gibt es Bemühungen in der Forschung und Entwicklung, PPS-Systeme auch für die Auftragsfertiger zu entwickeln. Die PPS-Lösungen für Auftragsfertiger sind vorwiegend in Deutschland entstanden.

Ein wichtiger Schritt auf diesem Weg ist z. B. das Loslösen von bestimmten Plausibilitätsprüfungen konventioneller PPS-Systeme. So muß es möglich sein, die Erzeugnisstrukturdaten zu bearbeiten ohne vorher im Artikelstamm das Erzeugnis angelegt zu haben. Der Auftragsfertiger erwartet, daß PPS-Systeme mindestens folgende Vorgehen unterstiitzen:
(1) Anlegen von Artikeldaten in verkürzter und unvollständiger Form,

(2) Anlegen von Erzeugnisstrukturen im Dialog,

(3) Anlegen von Erzeugnisstruktur ohne vorheriges Anlegen von Teilestammdaten,

(4) Ergänzen von unvollständigen Teilestammdaten,

(5) Umgang mit Teilestammdaten unterschiedlichen Vollständigkeitsgrades.

Für bestimmte Probleme der Auftragsfertiger existieren bereits praxisrelevante Teillösungen in PPS-Systemen, für die meisten der erhobenen Anforderungen gibt es jedoch noch keine marktrelevanten Lösungen.

\section{Verfasser}

Prof. Dr. oec. László Ungvári

Fachbereich Ingenieurwesen/Wirtschaftsingenieurwesen Technische Fachhochschule Wildau

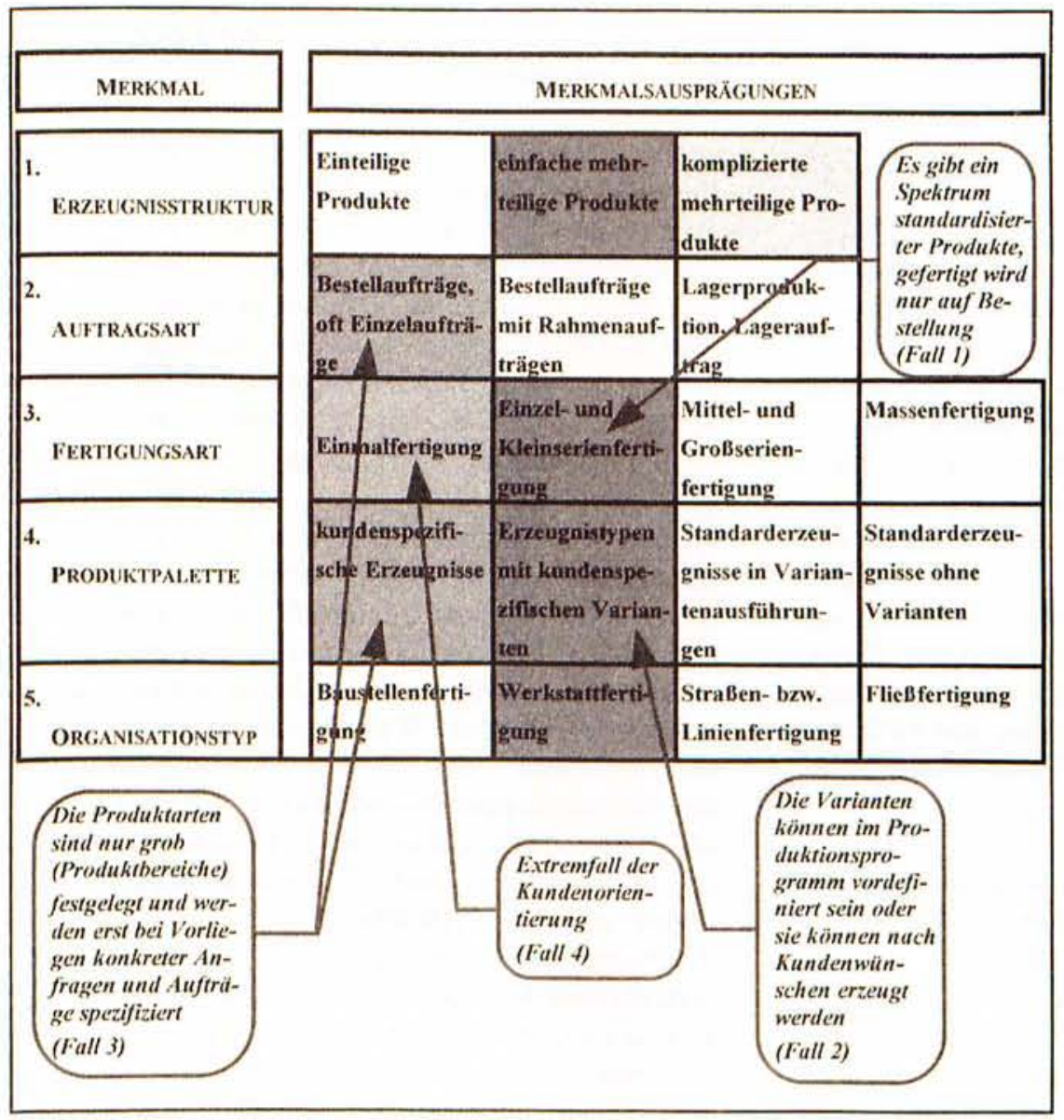

